\title{
NONEXISTENCE OF EQUIVARIANT DEGREE ONE MAPS
}

\author{
JANEY ANTONIO DACCACH
}

(Communicated by Haynes R. Miller)

\begin{abstract}
We establish the nonexistence of equivariant maps between some classes of manifolds carrying $G$-free actions and spheres, whose degree is prime to $|G|$, the order of $G$, where $G$ is a finite nontrivial group.
\end{abstract}

Dold, in his article [D], proved the following theorem:

If a map $f: S^{m} \rightarrow S^{n}$ commutes with some free action of a finite nontrivial group $G$ on the spheres $S^{m}, S^{n}$, then $n \geq m$.

We shall prove that this result remains true for all $G$-free actions on closed oriented manifolds and $G$-maps $f: M^{m} \rightarrow N^{n}$ under the following hypothesis:

Assume that $S^{m}$ has a free $G$-action and that there is a $G$-map $h: M^{m} \rightarrow S^{m}$ such that the degree of $h$ is prime to $|G|$.

We use the notion of the trace of an action.

For all $G$-spaces $X$, Gottlieb [G] defined an integer $\operatorname{tr}(G, X)$, called the trace of the action, having several properties. For our purposes, we select the following:

$G_{1}$ : If $G$ is a finite group of order $|G|$ acting on a manifold $N$ which is dominated by a finite CW complex, then $\operatorname{tr}(G, N)=|G|$ if and only if the action is free.

$G_{2}$ : If $M^{m}$ and $N^{m}$ are closed oriented compact $G$-manifolds where $G$ acts by orientation preserving homeomorphisms, and $f, g: M^{m} \rightarrow N^{m}$ are $G$-maps, then $\operatorname{tr}(G, M)$ divides the coincidence number $L(f, g)$.

In fact, if $\operatorname{deg} f$ or $\operatorname{deg} g$ is not zero, $G_{2}$ can be generalized as follows.

PROPOSITION 1. If $M^{m}$ and $N^{m}$ are closed oriented compact $G$-free manifolds and $f, g: M \rightarrow N$ are equivariant maps such that $\operatorname{deg} f \neq 0$ or $\operatorname{deg} g \neq 0$, then $\operatorname{tr}(G, M)$ divides $L(f, g)$.

PROOF. If $G$ acts by orientation preserving homeomorphisms then the result is nothing but $G_{2}$, proved by Gottlieb [G].

Now suppose that $\operatorname{deg} f \neq 0$. So if $h \in G$ reverses orientation in $M$, then $h$ also reverses orientation in $N$.

Let us define an action $G \times S^{1} \rightarrow S^{1}$ by

$$
\begin{cases}h(z)=\bar{z} & \text { if } h \text { reverses orientation, } \\ h(z)=z & \text { if } h \text { preserves orientation, }\end{cases}
$$

Received by the editors July 29, 1986.

1980 Mathematics Subject Classification (1985 Revision). Primary 55M25, 55M20; Secondary $57 \mathrm{~S} 17$.

Key words and phrases. Coincidence number, degree of a map, trace of an action, $G$-manifolds. 
for $h \in G$ and $z \in S^{1}$, the unit circle in the complex plane, and consider the $G$-free manifolds $M \times S^{1}$ and $N \times S^{1}$, and the equivariant maps

$$
f \times \text { id, } \quad g \times c: M \times S^{1} \rightarrow N \times S^{1}
$$

where the action of $G$ on $M \times S^{1}$ and $N \times S^{1}$ is the diagonal action, id is the identity function, and $c$ is the constant function, whose image is $1 \in S^{1}$.

Since $M \times 1 \subset M \times S^{1}$ is an equivariant retract it follows that $\operatorname{tr}\left(G, M \times S^{1}\right)=$ $\operatorname{tr}(G, M)$ (see $[\mathbf{G}]$ ); also since $G$ acts freely by orientation preserving homeomorphisms in $M \times S^{1}$ and $N \times S^{1}$ it follows from $G_{2}$ that $\operatorname{tr}\left(G, M \times S^{1}\right)$ divides $L(f \times$ id, $g \times c)$.

But an easy computation shows that $L(f \times$ id, $g \times c)=L(f, g)$. So $\operatorname{tr}(G, M)$ divides $L(f, g)$. Q.E.D.

Since in this note we assume that all manifolds are closed, compact, oriented, connected $G$-manifolds, where $G$ acts freely it follows that if $f, g: M^{m} \rightarrow N^{m}$ are $G$-maps, then $|G|$ divides $L(f, g)$. This result was also obtained by Nakaoka [N, Theorem 4.1].

From the definition of $L(f, g)$ (see [V]), it follows that if $f, g: M^{m} \rightarrow S^{m}$ are maps, then $L(f, g)=\operatorname{deg} f+(-1)^{m} \operatorname{deg} g$.

Proposition 2. Let $M^{m}$ and $N^{n}$ be $G$-free manifolds and let $M^{m}$ satisfy the hypothesis (H). If $f: M^{m} \rightarrow N^{n}$ is a $G$-map then $n \geq m$.

ProOF. Let us assume that $m>n$. Since we assume that $S^{m}$ is a $G$-free manifold, then $S^{m}$ is $m$-universal for the group $G$, because it is $(m-1)$-connected [St]. So there is a $G$-map $h_{1}: N^{n} \rightarrow S^{m}$. By hypothesis (H) we have also a $G$-map $h: M^{m} \rightarrow S^{m}$ whose degree is prime to $|G|$. Then we have two $G$ maps $\left(h_{1} \circ f\right), h: M^{m} \rightarrow S^{m}$ and $|G|$ divides $L\left(h_{1} \circ f, h\right)$. But $L\left(h_{1} \circ f, h\right)=$ $\operatorname{deg}\left(h_{1} \circ f\right)+(-1)^{m} \operatorname{deg} h$ and since $h_{1} \circ f$ factors through a manifold of lower dimension we have that $\operatorname{deg}\left(h_{1} \circ f\right)=0$; we conclude that $|G|$ divides $\operatorname{deg} h$.

This implies $G$ is trivial. Q.E.D.

Suppose now that the nontrivial finite group $G$ acts freely on the closed oriented manifolds $M^{n}, N^{n}, S^{m+n}$.

Let us consider the diagonal action of $G$ on the product $M^{m} \times N^{n}$. Since the projection to the first factor $\pi: M^{m} \times N^{n} \rightarrow M^{m}$ is equivariant, there is no equivariant map $f: M^{m} \times N^{n} \rightarrow S^{m+n}$ whose degree is prime to $|G|$. In particular we have

COROLLARY 3. If the nontrivial finite group $G$ acts freely on the closed oriented manifolds $M^{m}, N^{n}, S^{m+n}$, then there is no degree one equivariant map $f: M^{m} \times$ $N^{n} \rightarrow S^{m+n}$, where $G$ acts diagonally on $M \times N$.

As another application let us consider the Stiefel manifold $V_{k}\left(R^{n}\right)$, the set of $k$-orthonormal frames of $R^{n}$, and the involution $\alpha: V_{k}\left(R^{n}\right) \rightarrow V_{k}\left(R^{n}\right)$ defined by $\left(e_{i_{1}}, \ldots, e_{i_{k}}\right)=\left(-e_{i_{1}}, \ldots,-e_{i_{k}}\right)$.

Consider also the involution on $S^{m}$ given by the antipodal map.

COROLlaRY 4. For $k>1$ there is no equivariant odd degree map $f: V_{k}\left(R^{n}\right) \rightarrow$ $S^{m}$, where $m=\operatorname{dim} V_{k}\left(R^{n}\right)$.

PROOF. For $j<k$ the projection $\pi: V_{k}\left(R^{n}\right) \rightarrow V_{j}\left(R^{n}\right)$ is an equivariant map. Since $\operatorname{dim} V_{j}\left(R^{n}\right)<\operatorname{dim} V_{k}\left(R^{n}\right)$ the hypothesis (H) can not be satisfied. Q.E.D. 


\section{REFERENCES}

[D] Albrecht Dold, Simple proofs of Borsuk-Ulam results, Contemp. Math., vol. 19, Amer. Math. Soc., Providence, R.I., 1983, pp. 65-69.

[G] Daniel H. Gottlieb, The trace of an action and the degree of a map, Trans. Amer. Math. Soc. 287 (1985), 419-429.

[N] Minoro Nakaoka, Coincidence Lefschetz number for a pair of fibre preserving maps, J. Math. Soc. Japan 32 (1980).

[St] Norman Steenrod, The topology of fibre bundles, Princeton Univ. Press, Princeton, N.J., 1951.

[V] James W. Vick, Homology theory, Academic Press, New York, 1973.

ICMSC-USP, Caixa Postal 668, 13560-SÃo Carlos-SP, BRASIL 